\title{
ESTUDO DA DEGRADAÇÃO FOTOCATALÍTICA DE DIMETIL DISSULFETO: AVALIAÇÃO ESTATISTICA DO EFEITO DO PH DA TEMPERATURA E CONCENTRAÇÃO DO CONTAMINANTE ORGẨICO
}

\author{
Rogério Marcos Dallago*, Fabrício Domenech, Joncimar Reolon e Marco Di Luccio \\ Centro Tecnológico, Universidade Regional Integrada do Alto Uruguai e Missões, Campus Erechim, Av. Sete de Setembro, 1621, \\ 99700-000 Erechim - RS, Brasil \\ Silvia Maria da Silva Egues \\ Instituto de Tecnologia e Pesquisa, Universidade Tiradentes, Av. Murilo Dantas, 300, 149032-490 Aracaju - SE, Brasil
}

Recebido em 26/2/08; aceito em 10/9/08; publicado na web em 26/1/09

\begin{abstract}
ASSESSMENT OF THE PHOTOCATALYTIC DEGRADATION OF DIMETHYL DISULFIDE: EFFECT OF pH, TEMPERATURE AND CONCENTRATION OF THE ORGANIC CONTAMINANT. This work aimed to assess the photocatalytic degradation of a model odor compound (dimethyl disulfide, DMDS), found in liquid and gaseous wastes of plants for processing poultry byproducts. The effect of $\mathrm{pH}$ and temperature on adsorption and photocatalytic degradation was evaluated through factorial experimental designs. The results suggest the presence of an optimum region for adsorption, at $45.0^{\circ} \mathrm{C}$ and $\mathrm{pH} 4.0$. In the photocatalytic runs an optimum for temperature and $\mathrm{pH}$ was also observed. At $45{ }^{\circ} \mathrm{C}$ and $\mathrm{pH} 4.0$ the removal of DMDS was $99 \%$ after 60 min of irradiation.
\end{abstract}

Keywords: photocatalytic degradation; dimethyl disulfide; wastes.

\section{INTRODUÇÃO}

A poluição tem sido um assunto de extremo interesse em todas as partes do mundo. A crescente preocupação com o meio ambiente tem como principal conseqüência a criação de normas e legislações cada vez mais restritivas quanto à disposição final e ao descarte de subprodutos na biosfera. ${ }^{1}$

As atividades industriais vinculadas ao beneficiamento de alimentos, mais especificamente em fábricas de subprodutos de processamento de aves, caracterizam-se pela emissão e dispersão no ambiente de uma variedade de compostos sulfurados voláteis, como gás sulfídrico $\left(\mathrm{H}_{2} \mathrm{~S}\right)$, dimetil sulfeto (DMS, $\mathrm{Me}_{2} \mathrm{~S}$ ), dimetil dissulfeto (DMDS, $\mathrm{Me}_{2} \mathrm{~S}_{2}$ ), dimetil trissulfeto (DMTS, $\mathrm{Me}_{2} \mathrm{~S}_{3}$ ), metanotiol (MeSH) e dissulfeto de carbono ( $\mathrm{CS}_{2}$ ), os quais, dependendo da concentração, podem apresentar elevada toxicidade ao meio ambiente..$^{2-4}$

Esses compostos são produzidos por degradação biológica ou por decomposição térmica. Pelo fato desses sulfurados possuírem um baixo limiar de odor (concentração mínima detectável pelo olfato humano), a ocorrência dessas substâncias em emissões industriais pode causar sérios problemas de odores para essas empresas. A biodegradação de compostos odoríferos muitas vezes é ineficiente devido ao fato dos microrganismos consumirem preferencialmente algumas substâncias, deixando que outras passem pelo processo sem serem degradadas. ${ }^{5}$

Os processos oxidativos avançados (POAs) têm sido muito investigados e propostos como alternativa a processos convencionais, com especial atenção à fotocatálise heterogênea utilizando $\mathrm{TiO}_{2}$ como catalisador. ${ }^{6-8} \mathrm{~A}$ grande vantagem da fotocatálise heterogênea deve-se ao fato de que, na maioria das vezes, pode conduzir à total mineralização de compostos orgânicos. Desta forma, evita-se a transferência do poluente da fase aquosa para a sólida, além de aliar alta eficiência a baixos custos operacionais. ${ }^{9}$

Nesse sentido, este trabalho teve como objetivo avaliar a influência do $\mathrm{pH}$ e da temperatura sobre a eficiência da fotocatálise heterogênea na degradação de dissulfeto de dimetila (DMDS), utilizando

*e-mail: dallago@uricer.edu.br dióxido de titânio como fotocatalisador. O dissulfeto de dimetila foi escolhido como modelo, pois foi um dos compostos identificados em efluentes gasosos e águas de lavagem de gases, geradas em fábrica de subprodutos de processamento de carne de aves. ${ }^{10}$

\section{PARTE EXPERIMENTAL}

Fotocatalisador e composto odorífero modelo

$\mathrm{O} \mathrm{TiO}_{2}$ comercialmente disponível (Degussa P-25) foi utilizado como fotocatalisador sem qualquer tratamento adicional. Apresentase principalmente na forma anatásio $(80 \%$ anatásio e $20 \%$ rutilo em massa) e possui área superficial BET de $50 \mathrm{~m}^{2} \mathrm{~g}^{-1}$ e tamanho de partícula de aproximadamente $20 \mathrm{~nm}$.

Para a realização dos experimentos foi utilizado um padrão comercial do composto dissulfeto de dimetila 99\% (DMDS, Aldrich).

\section{Ensaios de adsorção e fotocatálise}

Os experimentos de adsorção e fotocatálise foram conduzidos em reator de vidro encamisado sem tampa, acoplado a um banho termostático ajustado para manter a temperatura constante. A agitação constante foi assegurada por um agitador magnético. $\mathrm{O}$ diâmetro e altura internos do reator são, respectivamente, 4,5 e $9,5 \mathrm{~cm}$, com volume útil de $100 \mathrm{~mL}$. Este sistema foi montado no interior de uma caixa de madeira com dimensões internas de 85 x 42,5 x 42,5 cm com paredes internas revestidas em papel alumínio. Os ensaios de adsorção foram realizados em batelada, em ambiente isento de luz, por $24 \mathrm{~h}$. As soluções foram mantidas sob agitação para promover uma completa homogeneização do catalisador. Periodicamente foram retiradas alíquotas de $2 \mathrm{~mL}$ do meio reacional, que foram centrifugadas e filtradas em membrana de éster de celulose $(0,22 \mu \mathrm{m}$, Millipore). A concentração do DMDS remanescente em solução foi determinada em espectrofotômetro UV/Vis (Agilent 8453) em um comprimento de onda de $205 \mathrm{~nm}$ utilizando cubeta de quartzo. As quantidades de substrato orgânico adsorvido no sólido foram determinadas por balanço de massa. 
Para os estudos fotocatalíticos, a irradiação na faixa do ultravioleta foi fornecida por uma lâmpada de vapor de mercúrio de $125 \mathrm{~W}$, marca Phillips (modelo HPLN 125W-IMP, fluxo luminoso $6200 \mathrm{~lm}$ e eficiência luminosa $50 \mathrm{~lm} / \mathrm{W}$ ), sem bulbo de vidro, posicionada a 50 $\mathrm{cm}$ da superfície do líquido. Os ensaios de adsorção foram conduzidos no mesmo sistema com a lâmpada apagada.

Os dados foram tratados utilizando o programa Statistica 6.0 (Statsoft Inc., Tulsa, OK, USA, www.statsoft.com). Foram realizadas réplicas de todos os experimentos, sendo que o erro experimental não ultrapassou a margem de $10 \%$.

\section{Efeito do pH e da temperatura na adsorção}

Para o estudo da adsorção, a influência de algumas variáveis foi investigada utilizando-se a técnica do planejamento de experimentos. ${ }^{11,12}$ Primeiramente, foi realizado um planejamento fatorial completo $2^{2}$ com três pontos centrais, para avaliação dos efeitos do $\mathrm{pH}$ e da temperatura na adsorção do DMDS em $\mathrm{TiO}_{2}$ (Tabela 1). Baseado nos resultados do primeiro planejamento elaborou-se um segundo planejamento fatorial $2^{2} \mathrm{com}$ triplicata do ponto central e dois pontos axiais para cada variável independente (planejamento composto central rotacional - PCCR, Tabela 1), avaliando-se os efeitos do $\mathrm{pH}$ e temperatura, fixando-se a concentração do DMDS $\left(2,5 \times 10^{-4} \mathrm{gmol} \mathrm{L}^{-1}\right)$. A faixa de estudo das variáveis foi determinada com base em ensaios anteriores. ${ }^{10}$

Tabela 1. Níveis codificados e valores reais das variáveis estudadas para os ensaios de adsorção e fotocatálise

\begin{tabular}{lcc}
\hline \multicolumn{2}{l}{ Planejamento Fatorial Completo $2^{2}$} & \\
\hline Níveis & Temperatura $\left({ }^{\circ} \mathrm{C}\right)$ & $\mathrm{pH}$ \\
\hline-1 & 22,6 & 3,4 \\
0 & 33,8 & 7,0 \\
+1 & 45,0 & 10,5 \\
\hline Planejamento Composto Central Rotacional (PCCR) \\
\hline Níveis & Temperatura $\left({ }^{\circ} \mathrm{C}\right)$ & $\mathrm{pH}$ \\
\hline$-1,41$ & 25,0 & 1,5 \\
-1 & 31,0 & 2,2 \\
0 & 45,0 & 4,0 \\
+1 & 59,0 & 5,8 \\
1,41 & 65,0 & 6,5 \\
\hline
\end{tabular}

As mesmas variáveis, faixas e planejamentos, foram investigadas nos estudos fotocatalíticos.

Após a realização dos planejamentos foi definida a melhor condição de pH e temperatura para a degradação fotocatalítica do DMDS. A partir das condições otimizadas avaliou-se o efeito da concentração de DMDS na faixa de $1,0 \times 10^{-4}$ a $1,0 \times 10^{-3} \mathrm{~mol} \mathrm{~L}^{-1}$.

Ensaios em branco, conduzidos sem a utilização de $\mathrm{TiO}_{2}$, foram realizados para se avaliar a magnitude da contribuição da fotodegradação e perdas por evaporação do DMDS, nos resultados obtidos para os ensaios conduzidos na presença de fotocatalisador $\left(\mathrm{TiO}_{2}\right)$.

\section{RESULTADOS}

\section{Fotólise}

A Figura 1 apresenta os resultados obtidos para os ensaios de fotodegradação conduzidos na ausência de $\mathrm{TiO}_{2}$.

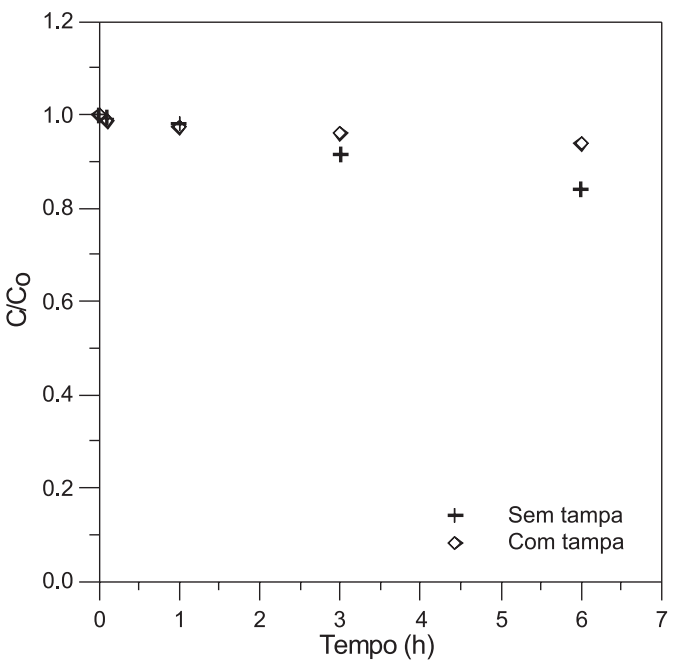

Figura 1. Variação da concentração de DMDS com o tempo para o ensaio de fotólise. Condições de operação: $\mathrm{pH}$ livre, $[D M D S]=2,5 \times 10^{-4} \mathrm{~mol} / \mathrm{L}$, $\left[\mathrm{TiO}_{2}\right]=0, \mathrm{~T}=25^{\circ} \mathrm{C}$

A taxa de remoção de DMDS, após 6 h de incidência, oscilou entre 10 e $20 \%$. A menor taxa de remoção observada para o ensaio conduzido com o reator tampado pode estar vinculada à propriedade que o vidro apresenta em absorver radiação na faixa do ultravioleta, principalmente acima de $300 \mathrm{~nm}$, limitando a radiação incidente sobre a solução. Constatou-se também que a presença da tampa contribui, minimizando perdas por volatilização do DMDS da solução.

\section{Efeito do pH e da temperatura na adsorção}

A adsorção dos poluentes na superfície do fotocatalisador é um dos principais pré-requisitos para a eficiência das reações fotocatalíticas. ${ }^{13,14}$ A Figura 2 apresenta as cinéticas da adsorção do dimetil dissulfeto (DMDS) para os experimentos do primeiro planejamento completo $2^{2}$ (Tabela 1). Pode-se observar que, independentemente das condições experimentais avaliadas, todos os ensaios apresentaram o mesmo comportamento, isto é, uma queda progressiva da concentração do DMDS na solução.

As maiores frações adsorvidas $(\approx 0,7)$ correspondem aos ensaios conduzidos com o menor valor de $\mathrm{pH}(3,4)$ e diferentes níveis de tem-

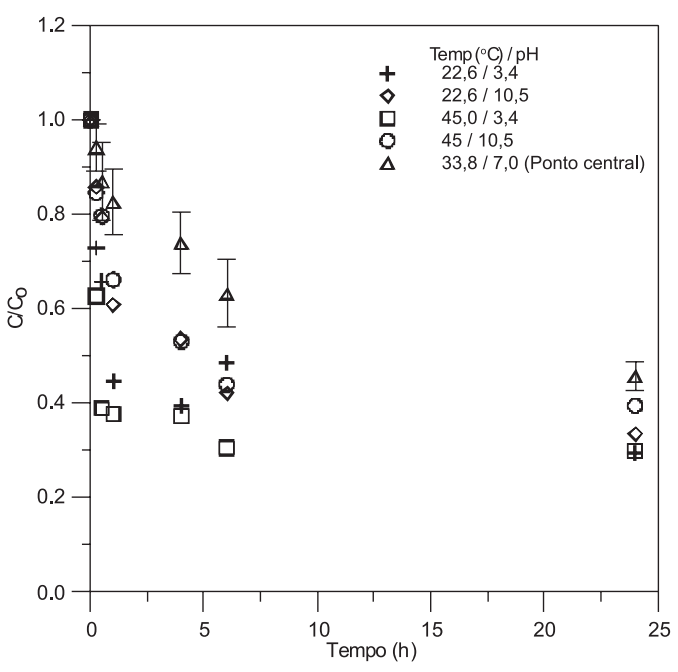

Figura 2. Variação da concentração de DMDS com o tempo para os experimentos do primeiro planejamento completo $2^{2}$ 
peratura. O tratamento estatístico (software Statistica 6.0) dos valores referentes às frações adsorvidas após $24 \mathrm{~h}$ de contato demonstrou que todas as variáveis ( $\mathrm{pH}$, temperatura e $\mathrm{pH}$ x temperatura) apresentaram efeito negativo significativo $(\mathrm{p}<0,05)$ sobre o processo adsortivo.

$\mathrm{O}$ efeito do $\mathrm{pH}$ no ensaio adsortivo pode ser explicado principalmente pela carga da superfície do $\mathrm{TiO}_{2}$ (ponto de carga zero do $\mathrm{TiO}_{2} \mathrm{P}-25, \mathrm{pH}_{\mathrm{pcz}} \sim 6$ ), uma vez que alterações no $\mathrm{pH}$ modificam sua carga superficial. ${ }^{15,16} \mathrm{Em} \mathrm{pH}$ inferior ao ponto de carga zero (PCZ), o catalisador está com predominância de cargas positivas em sua superfície, favorecendo a adsorção, mediante interações eletrostáticas, do DMDS, que se caracteriza por apresentar elevada densidade eletrônica vinculada aos pares de elétrons livres dos átomos de enxofre.

Em relação à temperatura, seu efeito está diretamente vinculado às propriedades termodinâmicas da interação adsorvato/adsorvente. Dependendo do poluente e do adsorvato envolvidos no processo adsortivo, o mesmo pode se caracterizar como endotérmico ou exotérmico. Por exemplo, a adsorção de chumbo, mediante interações eletrostáticas apresenta-se como um processo endotérmico quando a argila é empregada como adsorvente. No entanto, quando a adsorvente for a kaolinita ou a montmorilonita o processo é caracterizado como exotérmico. ${ }^{17}$ Neste estudo, o efeito negativo observado para a temperatura sugere ser o processo adsortivo exotérmico, apresentando um aumento da capacidade adsortiva de DMDS com a diminuição da temperatura. Além disto, o aumento da temperatura, por favorecer a solubilidade do soluto aumentando sua solvatação, limita sua atração para a superfície do adsorvente. ${ }^{17}$

Para o segundo planejamento experimental (PCCR - Tabela 1), conduzido empregando faixas de temperatura e $\mathrm{pH}$ ajustadas e ampliadas, as maiores frações adsorvidas após $14 \mathrm{~h}$ de contato foram observadas para as condições do ponto central (Tabela 2). O limite inferior da temperatura, mesmo esta apresentando efeito negativo, foi mantido à temperatura ambiente $\left(25^{\circ} \mathrm{C}\right)$ por questões de economia e praticidade, visando a aplicação deste processo em escala industrial.

A análise estatística dos resultados deste segundo planejamento forneceu o gráfico de Pareto (Figura 3), onde somente os efeitos de segunda ordem foram estatisticamente significativos $(p<0,05)$ e negativos. Isto é um indicativo de que há um ponto de ótimo para estas duas variáveis, o qual se encontra próximo à condição experimental do ponto central $\left(45,0{ }^{\circ} \mathrm{C}\right.$ e $\left.\mathrm{pH} 4,0\right) .{ }^{12}$

Tabela 2. Matriz do segundo planejamento experimental $2^{2}$ completo (valores codificados e reais) para avaliação da temperatura e $\mathrm{pH}$ ótimos, com as respostas em termos da fração adsorvida após $14 \mathrm{~h}$ de contato

\begin{tabular}{lccc}
\hline Ensaio & Temperatura $\left({ }^{\circ} \mathrm{C}\right)$ & $\mathrm{pH}$ & Fração adsorvida $14 \mathrm{~h}$ \\
\hline 1 & $-1(31,0)$ & $1(5,8)$ & 0,638 \\
2 & $1(59,0)$ & $1(5,8)$ & 0,678 \\
3 & $-1(31,0)$ & $-1(2,2)$ & 0,647 \\
4 & $1(59,0)$ & $-1(2,2)$ & 0,664 \\
5 & $0(45,0)$ & $1,41(6,5)$ & 0,626 \\
6 & $0(45,0)$ & $-1,41(1,5)$ & 0,606 \\
7 & $1,41(65,0)$ & $0(4,0)$ & 0,556 \\
8 & $-1,41(25,0)$ & $0(4,0)$ & 0,679 \\
9 & $0(45,0)$ & $0(4,0)$ & 0,728 \\
10 & $0(45,0)$ & $0(4,0)$ & 0,717 \\
11 & $0(45,0)$ & $0(4,0)$ & 0,679 \\
12 & $0(45,0)$ & $0(4,0)$ & 0,684 \\
\hline
\end{tabular}

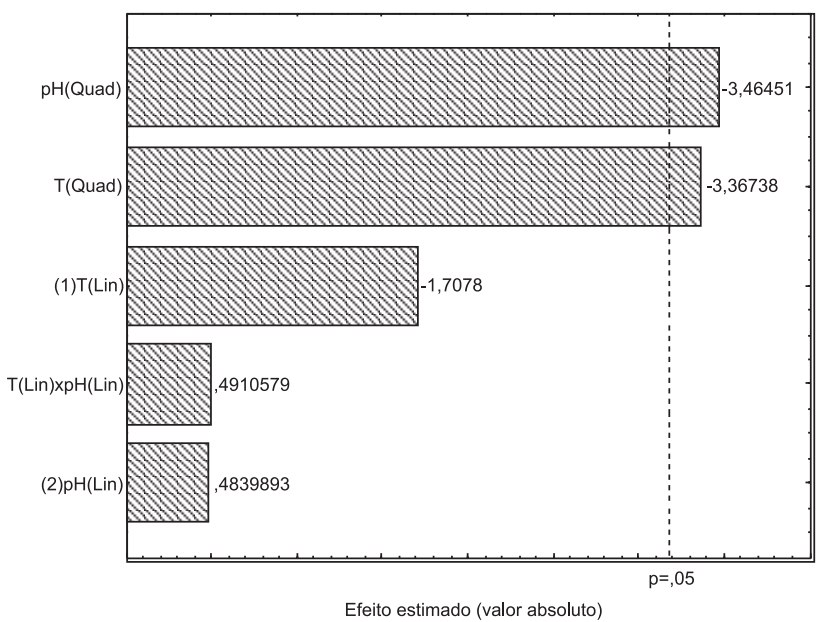

Figura 3. Gráfico de Pareto da fração adsorvida após 14 h de contato da solução de DMDS com o $\mathrm{TiO}_{2}$ em função do $\mathrm{pH}$ e da temperatura

\section{Fotocatálise}

O fluxo fotônico do sistema foi determinado seguindo a metodologia descrita por Kuhn et al. ${ }^{18} \mathrm{O}$ valor de fluxo fotônico obtido para 1 min de irradiação, $6,06 \times 10^{-5}$ einstein s ${ }^{-1}$, está coerente com valores descritos na literatura para este tipo de lâmpada. ${ }^{19}$

Previamente à etapa de fotocatálise, todos os ensaios foram submetidos a uma etapa de adsorção, empregando as mesmas condições experimentais $\left(25^{\circ} \mathrm{C}\right.$, sem controle de $\mathrm{pH}$, por um período de $14 \mathrm{~h}$ de contato solução/fotocatalisador). Posteriormente a este período, necessário para atingir o equilíbrio, as condições experimentais ( $\mathrm{pH}$ e temperatura), descritas no planejamento composto central rotacional (PCCR) $2^{2}$ - Tabela 1 , foram ajustadas, dando inicio à etapa fotocatalítica (Figura 4).

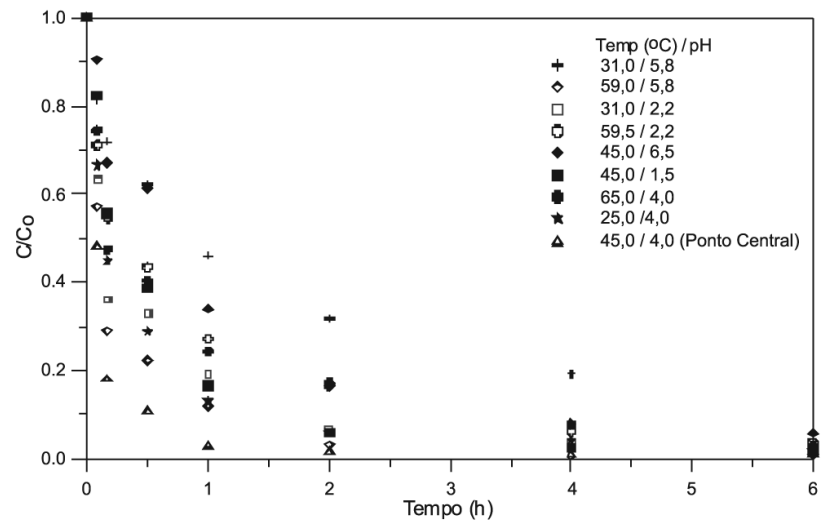

Figura 4. Variação da concentração de DMDS com o tempo para os experimentos do segundo planejamento completo $2^{2}$ com pontos axiais

Pode-se observar que, independente das condições experimentais empregadas, todos os ensaios apresentaram mesma tendência de degradação, apresentando em 6 h de irradiação uma remoção do DMDS presente na solução de aproximadamente $100 \%$.

As frações de DMDS removidos após 30 e 60 min de irradiação são apresentadas na Tabela 3 .

A análise estatística foi efetuada para os dois tempos (30 e 60 min), fornecendo os mesmos resultados. A Equação 1 apresenta o modelo codificado otimizado para a remoção fotocatalítica do DMDS, após 30 min de irradiação, o qual foi validado pela análise de variância apresentada na Tabela 4. 
Tabela 3. Matriz do segundo planejamento experimental $2^{2}$ completo (valores codificados e reais) com as respostas em termos da fração removida por fotocatálise após 30 e 60 min de exposição à fonte de irradiação

\begin{tabular}{lcccc}
\hline Ensaio & Temperatura $\left({ }^{\circ} \mathrm{C}\right)$ & $\mathrm{pH}$ & $\begin{array}{c}\text { Fração } \\
\text { removida } \\
30 \text { min }\end{array}$ & $\begin{array}{c}\text { Fração } \\
\text { removida } \\
60 \text { min }\end{array}$ \\
\hline 1 & $-1(31,0)$ & $1(5,8)$ & 0,775 & 0,834 \\
2 & $1(59,0)$ & $1(5,8)$ & 0,928 & 0,962 \\
3 & $-1(31,0)$ & $-1(2,2)$ & 0,884 & 0,933 \\
4 & $1(59,0)$ & $-1(2,2)$ & 0,854 & 0,909 \\
5 & $0(45,0)$ & $1,41(6,5)$ & 0,771 & 0,873 \\
6 & $0(45,0)$ & $-1,41(1,5)$ & 0,848 & 0,936 \\
7 & $1,41(65,0)$ & $0(4,0)$ & 0,822 & 0,892 \\
8 & $-1,41(25,0)$ & $0(4,0)$ & 0,907 & 0,958 \\
9 & $0(45,0)$ & $0(4,0)$ & 0,964 & 0,993 \\
10 & $0(45,0)$ & $0(4,0)$ & 0,959 & 0,987 \\
11 & $0(45,0)$ & $0(4,0)$ & 0,980 & 0,990 \\
12 & $0(45,0)$ & $0(4,0)$ & 0,969 & 0,993 \\
\hline
\end{tabular}

Tabela 4. Análise de variância para a remoção fotocatalítica de DMDS em 30 min

\begin{tabular}{lcccc}
\hline $\begin{array}{l}\text { Fontes de } \\
\text { variação }\end{array}$ & $\begin{array}{c}\text { Soma dos } \\
\text { Quadrados }\end{array}$ & $\begin{array}{c}\text { Graus de } \\
\text { Liberdade }\end{array}$ & $\begin{array}{c}\text { Quadrados } \\
\text { Médios }\end{array}$ & F calculado \\
\hline Regressão & 0,0518 & 4 & 0,0130 & 9,48 \\
Resíduos & 0,00957 & 7 & 0,00137 & \\
Falta de ajuste & 0,00934 & 4 & & \\
Erro puro & 0,000235 & 3 & & \\
Total & 0,0614 & 11 & & \\
\hline
\end{tabular}

Resíduos $=$ Falta de ajuste + erro puro. $\mathrm{F}_{0,95 ; 4 ; 7}=4,12$. Coeficiente de correlação: $R=0,92$

Remoção DMDS $=0,968-0,0458 \cdot \mathrm{T}^{2}-0,018 \cdot \mathrm{pH}-0,0735 \cdot \mathrm{pH}^{2}+$ 0,0459.T.pH

Verifica-se que o coeficiente de correlação obtido $(R=0,92)$ e o teste $F$ ( 2,3 vezes maior que o valor tabelado) validaram estatisticamente o modelo $(\mathrm{p}<0,05)$, permitindo a construção da superfície de resposta e curva de contorno (Figuras $5 \mathrm{a}$ e b).

Observa-se que, na faixa investigada, ambas variáveis independentes apresentaram efeito significativo sobre a degradação do DMDS. Verifica-se a presença de uma região de ótimo localizada em torno do ponto central $\left(\mathrm{pH}=4\right.$ e $\left.45^{\circ} \mathrm{C}\right)$, que está coerente com a literatura, que descreve uma região de temperatura ótima entre 20 e $80^{\circ} \mathrm{C}$, devido à pequena energia de ativação aparente..$^{20,21}$ Este comportamento está de acordo com os resultados de adsorção, que se caracteriza como principal etapa limitante do processo de fotodegradação. ${ }^{7,20} \mathrm{O}$ efeito do $\mathrm{pH}$, no que se refere à degradação fotocatalítica, atua efetivamente na etapa adsortiva. Em $\mathrm{pH}$ maior que o ponto de carga zero (PCZ), o catalisador está com predominância de cargas negativas em sua superfície, desfavorecendo a adsorção e degradação do DMDS. Por outro lado, $\mathrm{pH}$ excessivamente baixo leva à redução da taxa de degradação, uma vez que a adsorção completa do DMDS pode ocorrer, bloqueando os sítios ativos do $\mathrm{TiO}_{2}$, impedindo a ativação fotônica dos mesmos e, conseqüentemente, a a)
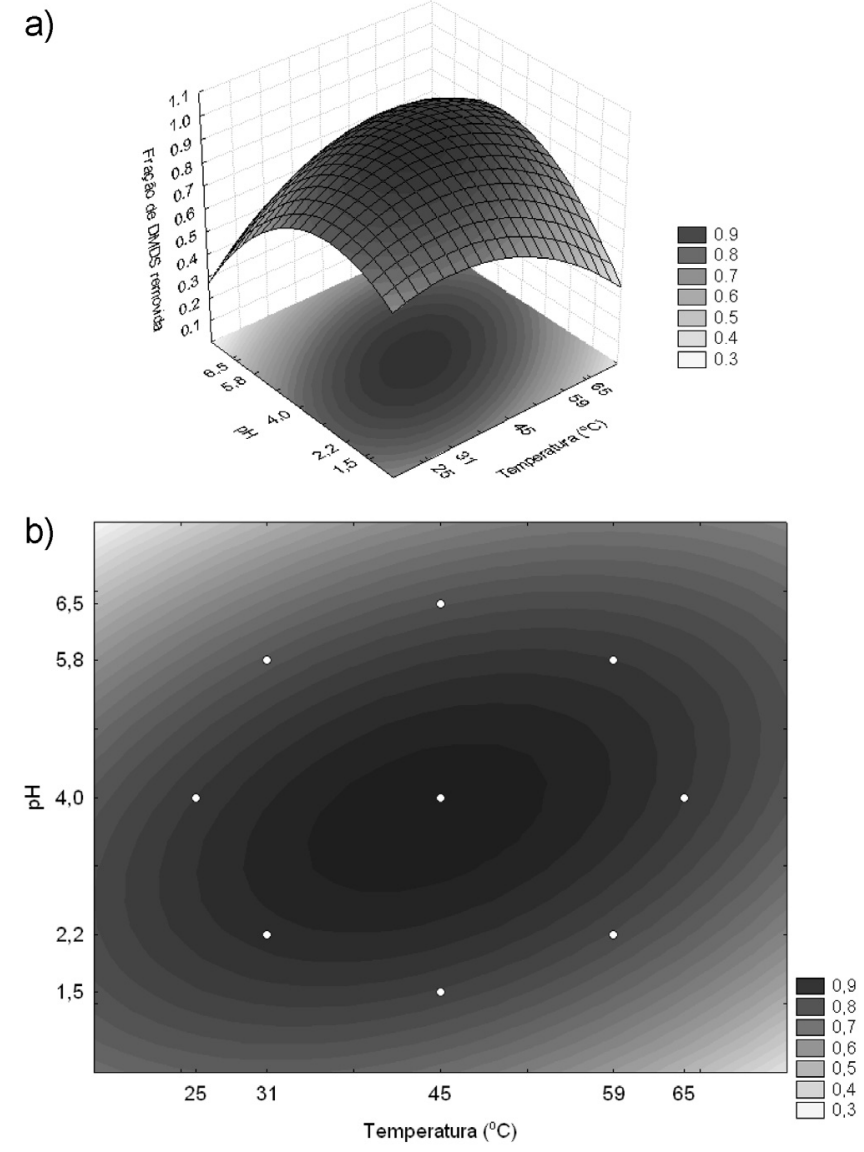

Figura 5. Superfície de resposta (a) e curva de contorno (b) para a fração de $D M D S$ removida em função do $\mathrm{pH}$ e da temperatura em 30 min de exposição à fonte de irradiação

degradação fotocatalítica. Ainda, o pH baixo pode levar à aglomeração das partículas de $\mathrm{TiO}_{2}$, diminuindo a área superficial para adsorção e absorção de fótons. ${ }^{15,16}$

$\mathrm{O}$ pH também pode afetar o mecanismo de degradação. Enquanto em $\mathrm{pH}$ baixo as lacunas $\left(\mathrm{h}^{+}\right)$desempenham um papel predominante na oxidação, em pH neutro a alcalino os radicais hidroxila são as espécies oxidantes predominantes, pela facilidade na sua geração devido à maior presença de íons hidróxido disponíveis na superfície do catalisador. No entanto, $\mathrm{pH}$ elevado pode levar à repulsão entre a superfície do catalisador e a molécula de DMDS, por exclusão de cargas, e entre a superfície do catalisador e os íons hidroxila, diminuindo a taxa de formação dos radicais hidroxila. ${ }^{22}$ Neste contexto, o efeito do $\mathrm{pH}$ dependerá da magnitude com que cada um destes mecanismos contribui em cada sistema estudado, sugerindo a existência de um pH ótimo para a fotodegradação, de acordo com o que foi observado.

As variações de temperatura e pH na região de máxima conversão sugerem que o efeito da temperatura foi menos pronunciado que o do $\mathrm{pH}$ (Figura 5b).

Gálves et al. ${ }^{23}$ mostraram que a velocidade das reações fotoquímicas não sofre grande influência da temperatura, o que é um comportamento típico das reações iniciadas por absorção de fótons. Rigoni, ${ }^{24}$ estudando a degradação fotocatalítica de corante alimentício, observou também um efeito pouco pronunciado da temperatura.

Considerando que a variação de temperatura influencia diretamente na velocidade com que as reações se processam (a velocidade aumenta proporcionalmente com a temperatura) e no equilíbrio químico das mesmas (processo exotérmico ou endotérmico), a ocorrência 
de uma faixa ótima de temperatura sugere um efeito competidor entre o aumento das taxas de reação e a diminuição da capacidade de adsorção com o aumento da temperatura.

\section{Efeito da concentração de DMDS na degradação fotocatalítica}

O efeito da concentração de DMDS na cinética de degradação não foi incluído no planejamento de experimentos, uma vez que poderia mascarar o efeito das outras variáveis independentes, pois o efeito de concentração em cinéticas de degradação normalmente é negativo. ${ }^{24}$

Os resultados referentes a este estudo, apresentados na Figura 6, demonstram que mesmo em uma ampla faixa de concentração (1,0x $10^{-4}$ a $1,0 \times 10^{-3} \mathrm{~mol} \mathrm{~L}^{-1}$ ou 9,4 a $\left.94 \mathrm{mg} \mathrm{L}^{-1}\right)$ é possível remover mais de $90 \%$ da concentração inicial de DMDS após $6 \mathrm{~h}$ de irradiação. Como esperado, o aumento da concentração de DMDS, mantendo a quantidade de catalisador fixa, leva a uma menor taxa de degradação. Este efeito é comum em processos com catálise heterogênea, pois como o mecanismo envolve a adsorção dos compostos a serem degradados sobre a superfície do catalisador, em concentrações mais elevadas é possível que todos os sítios catalíticos estejam ocupados, limitando o processo de adsorção/degradação.

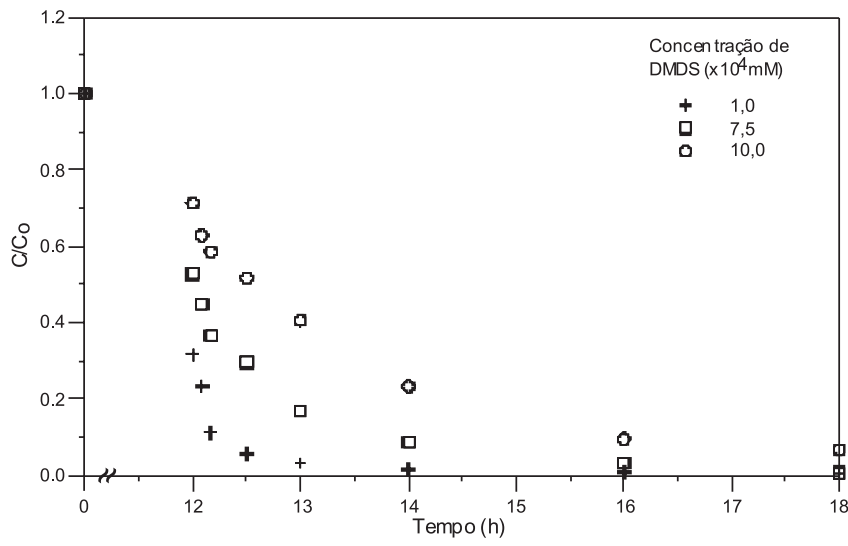

Figura 6. Variação da concentração de DMDS com o tempo para diferentes

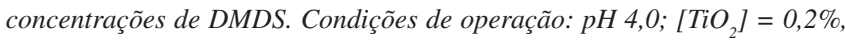
$T=25^{\circ} \mathrm{C}$

A linearização dos resultados da Figura 6, considerando-se um modelo cinético de pseudo primeira ordem, encontra-se apresentada na Tabela 5.

Tabela 5. Constantes de taxas iniciais de reação e tempos de meia vida para degradação fotocatalítica do DMDS utilizando $\mathrm{TiO}_{2}$

\begin{tabular}{lcccc}
\hline $\mathrm{C}_{\mathrm{o}}\left(\mathrm{x} 10^{4} \mathrm{~mol} \mathrm{~L}^{-1}\right)$ & $\mathrm{C}_{\mathrm{eq}}\left(\mathrm{x} 10^{4} \mathrm{~mol} \mathrm{~L}^{-1}\right)$ & $\mathrm{k}\left(\mathrm{min}^{-1}\right)$ & $\mathrm{t}_{1 / 2}(\mathrm{~min})$ & $\mathrm{R}^{2}$ \\
\hline 1,0 & 0,618 & 0,0570 & 12,2 & 0,96 \\
7,5 & 4,42 & 0,0177 & 39,1 & 0,97 \\
10 & 8,05 & 0,00857 & 81,0 & 0,92 \\
\hline
\end{tabular}

Os valores das constantes de taxa de reação e tempos de meiavida, encontrados para as diferentes concentrações de DMDS estudadas, são comparáveis aos obtidos por Demeestere et al. ${ }^{25}$ para a degradação de dimetil sulfeto (DMS) por fotocatálise em fase gasosa, utilizando $\mathrm{TiO}_{2}$ dopado com enxofre. No trabalho destes autores, o menor tempo de meia-vida obtido foi de 10 min para uma concentração de DMS de $109 \mathrm{mg} \mathrm{L}^{-1}$, enquanto que o maior foi de $74 \mathrm{~min}$ para uma concentração de equilíbrio de $539 \mathrm{mg} \mathrm{L}^{-1}$, utilizando uma fonte de irradiação na faixa do espectro visível.

\section{CONCLUSÕES}

Os resultados obtidos no planejamento fatorial mostraram que somente os efeitos de segunda ordem foram estatisticamente significativos $(p<0,05)$ e negativos, indicando a presença de uma região mais favorável à adsorção localizada próxima à condição experimental do ponto central $\left(45^{\circ} \mathrm{C}\right.$ e $\left.\mathrm{pH} 4,0\right)$.

Em relação aos ensaios fotocatalíticos, também se observou um ótimo de degradação para a região em torno do ponto central. Empregando uma temperatura de $45^{\circ} \mathrm{C}$ e pH 4,0 obteve-se uma remoção de DMDS da solução de aproximadamente $99 \%$ após 60 min de irradiação. $\mathrm{O}$ efeito do $\mathrm{pH}$ foi vinculado a alterações da carga na superfície do catalisador e da densidade eletrônica do DMDS.

A diminuição da taxa de remoção com o aumento da concentração de DMDS foi vinculada ao mecanismo envolvido no processo de adsorção do DMDS sobre a superfície do catalisador, a qual, com o aumento da concentração amplia a possibilidade de saturação dos sítios catalíticos, limitando o processo de adsorção/degradação.

\section{REFERÊNCIAS}

1. Jardim, W. F.; Fotocatálise: aplicações ambientais, Instituto de Química, UNICAMP, 1996.

2. Rappert, S.; Müller, S.; Waste Manage. 2005, 25, 887.

3. Cheremisinoff, P. N.; Air Pollution Control and Design for Industry, Marcel Dekker: New York, 1993.

4. Wright, D. W.; Eaton, D. K.; Nielsen, F. W. K.; Koziel, J. A.; Spinhirne, J. P.; Parker, D. B.; J. Agric. Food Chem. 2005, 53, 8663.

5. Zhang, L.; Hirai, M.; Shoda, M.; J. Ferment. Bioeng. 1991, 72, 392.

6. Nogueira, R. F. P.; Jardim, W. F.; Quim. Nova 1998, 21, 69.

7. Konstantinou, I. M.; Albanis, T. A.; Appl. Catal., B 2004, 49, 1.

8. Karkmaz, M.; Puzenat, E.; Guillard, J.; Herrmann, J. M.; Appl. Catal., B 2004, 51, 181

9. Poulios, I.; Tsachpinis, I.; J. Chem. Technol. Biotechnol. 1999, 74, 349.

10. Krüger, R. L.; Dissertação de Mestrado, Universidade Regional Integrada do Alto Uruguai e das Missões, Brasil, 2006.

11. Barros Neto, B.; Scarminio, I. S.; Bruns, R. E.; Planejamento e otimização de experimentos, $2^{\mathrm{a}}$ ed., UNICAMP: Campinas, 1996.

12. Rodrigues, M. I.; Iemma, A. F.; Planejamento de experimentos e otimização de processos: uma estratégia seqüencial de planejamento, Ed. Casa do Pão: Campinas, 2005.

13. Fox, M.; Dulay, M. T.; Chem. Rev. 1993, 93, 341.

14. Murugababdham, M.; Swaminathan, M.; Dyes Pigm. 2006, 68, 133.

15. Fernàndez, J.; Kiwi, J.; Baeza, J.; Freer, J.; Lizama C.; Mansilla, H. D.; Appl. Catal., B 2004, 48, 205.

16. Sauer, T. P.; Casaril, L.; Humeres, E.; Moreira, R. F. P. M.; J. Appl. Electrochem. 2005, 35, 821.

17. Bhattacharyya, K. G.; Gupta, S. S.; Colloids Surf., A 2006, 277, 191.

18. Kuhn, H. J.; Braslavsky, S. E.; Schmidt, R.; Pure Appl. Chem. 2004, 76, 2105.

19. Cesconetto Neto, G.; Sauer, T. P.; José, H. J.; Moreira, R. F. P. M.; Humeres, J. J. E.; J. Air Waste Manage. 2004, 54, 77.

20. Herrmann, J. M.; Catal. Today 1999, 53, 115.

21. Gogate, P. R.; Pandit, A. B.; Adv. Environ. Res. 2004, 8, 501.

22. Habibi, M. H.; Hassanzadeh, A.; Mahdavi, S.; J. Photochem. Photobiol., A 2005, 172, 89.

23. Gálvez, J. B.; Rodríguez, S. M.; Gasca, C. A. E.; Bandala, E. R.; Gelover, S.; Leal, T. Em Purificación de águas por fotocatálisis heterogénea: estado da arte; Miguel Blesa ed.; La Plata, 2001, cap. 3.

24. Rigoni, R. E.; Dissertação de Mestrado, Universidade Regional Integrada do Alto Uruguai e das Missões, Brasil, 2006.

25. Demeestere, K.; Dewulf, J.; Witte, B. D.; Langenhove, H. V.; Appl. Catal., B 2005, 60, 93. 